\title{
Incentives, subject matter and length of questionnaire affect response rates
}

Tan R T, Burke FJ T. Response rates to questionnaires mailed to dentists. A review of 77 publications. Int Dent J 1997; 47: 349-354

Objectives To investigate responses rates and factors affecting response rate of dentists to mailed questionnaires and to suggest methods of improving the response.

Data sources Hand search of the British Dental Journal, Journal of Prosthetic Dentistry, Journal of American Dental Association and Dental Update 1989-1992.

Study selection All papers including mailed questionnaires were included. (Some copies of JADA in 1992 were not searched).

Data extraction Various aspects of questionnaire design were noted and a simple subjective assessment of the length of the questionnaire into three categories was made (simple, moderate and complicated). Overall response rate was measured as was the status of the respondents and their country of origin.

Results Of the 3140 papers examined $2.5 \%$ (77) involved questionnaires. Response rates varied from 17-100\%. Simple questionnaires produce a higher response rate than complex ones
(60\% compared with $72 \%)$. Higher response rates were obtained from those carried out in Australia or Canada ( 3 studies only) and which were short in length (less than 10 questions or requiring no written answers). The use of various incentives such as reply paid envelopes, reminders, appears to increase response rate the highest responses being in those questionnaires with more incentives.

Conclusion The subject of the questionnaire, incentives offered and the length of the questionnaire may be factors involved in the response rates to mailed questionnaires. The authors suggest many methods for increasing response rates derived from a further review of the literature. Other possible factors include advanced notification, appearance and personalisation of the questionnaire, presence and wording of a covering letter asking for help and stressing the importance of the survey, confidentiality and well timed reminders.

Address F J T Burke, University of Glasgow Dental Hospital and School, 378 Sauchiehall Street, Glasgow G2 3JZ, UK

\section{Commentary}

The study by Tan and Burke is a review of published papers in four dental journals over a four year time period and is therefore retrospective. It is a very useful paper for those considering a mailed questionnaire. The objectives were to investigate the range and factors affecting response rates and suggest methods of improving response rates for questionnaires mailed to dentists.

It is very difficult to draw solid conclusions using a retrospective analysis of questionnaires as there is little standardisation of design and there are so many possible variables. More meaningful results might be obtained using a prospective, randomised design in which two sets of questionnaires are mailed differing in one variable only. However, it would take many studies to evaluate all the variables.

The authors identify a number of key ways to improve response rates. These include interest in the subject by the respondents, offering incentives such as reply paid envelopes, money, gifts and a promise to send the survey results. The design, appearance length and personalisation of the questionnaire are key factors. A user friendly covering letter asking for help and stressing the importance of the survey and its confidentiality with well timed reminders are all seen to be factors involved in response rates to mailed questionnaires.

The method by which the overall response rates are calculated is not described so there is uncertainty about consideration given to studies of differing size when making these calculations.

In the sense that science is knowledge ascertained by observation and experiment the results of questionnaires cannot be regarded as scientific as they rely upon subjects reporting behaviour or attitudes rather than observation by researchers. However, much valuable information can be gleaned from questionnaires regarding many issues such as attitude towards or uptake of guidelines and the use of services, materials or techniques.

\section{Malcolm Bruce}

Vocational Training Advisor and General Dental Practitioner, Devon, UK 